\title{
A New Decoupling Method for Explicit Stiffness Analysis of Kinematically Redundant Planar Parallel Kinematic Mechanism
}

\author{
Hyun-Pyo Shin ${ }^{1}$ and Donghun Lee ${ }^{2}$ \\ ${ }^{1}$ School of Robot and Automation Engineering, Dongyang Mirae University, Seoul 08221, Republic of Korea \\ ${ }^{2}$ School of Mechanical Engineering, Soongsil University, Seoul 07040, Republic of Korea
}

Correspondence should be addressed to Donghun Lee; dhlee04@gmail.com

Received 29 June 2015; Accepted 19 October 2015

Academic Editor: Zoran Gajic

Copyright (C) 2015 H.-P. Shin and D. Lee. This is an open access article distributed under the Creative Commons Attribution License, which permits unrestricted use, distribution, and reproduction in any medium, provided the original work is properly cited.

Optimization and control of stiffness for parallel kinematic mechanisms (PKM) are critical issues because stiffness is directly related to the precision and response characteristics of the end-effector of PKMs. Unlike nonredundant PKMs, redundant PKMs have additional actuators exceeding their essential degrees-of-freedom (DOF), resulting in an increase in the redundancy of control. The stiffness of redundant PKMs is divided into passive and active stiffness. Active stiffness is changeable even in cases of fixed kinematic parameters and end-effector posture. However, it is not easy and intuitive to control the active stiffness of redundant PKMs for the complexity of Hessian matrix operations. This paper describes a new decoupling method for explicit stiffness analysis of redundant PKM with the well-known two-DOF and one-redundant planar five-bar PKM. Three actuating joints are decoupled to three groups containing two actuating joints. With this mathematical configuration, the stiffness matrix for one-redundant actuation is also divided into three stiffness matrices for nonredundant actuation, and the contribution of each actuator can be intuitively investigated. Stiffness matrices for the original and decoupled cases are compared in detail. In particular, this decoupling method is applicable to redundant PKMs with many passive joints. Finding optimal joints for one- or two-redundant actuation with various candidates is more intuitive with this decoupling method.

\section{Introduction}

In many applications of parallel kinematic mechanisms (PKMs), the magnitude and isotropy of stiffness matrices are investigated as primary concerns. Stiffness is directly related to the precision of machining of machine tools and to the response characteristics of the end-effector of haptic devices. Optimization of stiffness, for example, maximizing stiffness in all directions [1], maximizing stiffness in one direction [2], securing a minimally required magnitude of stiffness, and increasing the isotropy of stiffness [3] are still critical issues according to the objectives of PKM applications.

Redundantly actuated PKMs have additional actuators exceeding their essentially required degrees-of-freedom (DOF), which increase the redundancy of control [4]. This feature serves as an advantage over nonredundant PKMs such as internal preload control for backlash avoidance, removing singularities to enlarge the usable workspace, and stiffness enhancements [5]. In designing the PKM for machine tools, the stiffness better be uniform with respect to any direction of external forces [6]. Through the appropriate torque distribution with respect to the position and orientation of the PKM, the total stiffness can be increased or decreased [7].

Stiffness of PKMs is divided into passive and active stiffness [8]. Passive stiffness is decided during the design process of the PKM and only changes according to the endeffector position and orientation if there is no additional changes in link length and actuator specifications such as torsional stiffness of motors and reducers. On the other hand, active stiffness can be controlled by the generation of internal preload torque distribution even though the design process is already finished. 


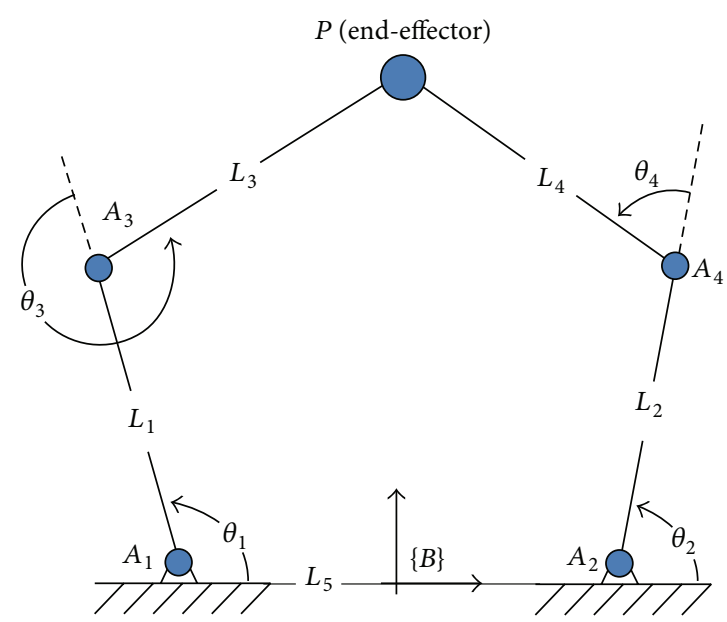

Figure 1: Kinematic structure for the planar five-bar redundant parallel kinematic mechanism.

Stiffness is investigated, characterized, and optimized for practical applications. Arsenault and Boudreau [9] introduced a reliable synthesis method capable of selecting optimal geometrical parameters of planar parallel mechanisms for considering workspaces, dexterity, stiffness, and singularity avoidance. Alici and Shirinzadeh [10] presented the enhanced stiffness modeling and analysis of robot manipulators and a methodology for their stiffness identification and characterization. Liu et al. [11] showed various optimization examples by applying global conditioning, global velocity, global payload, and global stiffness indexes. Lee et al. [12] performed kinematic and stiffness analyses including optimizations for maximizing active stiffness of the fivebar planar PKM. Kock and Schumacher [13] presented singularity configuration and stiffness analyses for 3-DOF redundantly actuated planar PKM and implemented the control for securing the minimum required stiffness. $\mathrm{Li}$ and $\mathrm{Xu}$ [14] investigated the stiffness behavior of the PKM via eigenscrew decomposition of the stiffness matrix. Pashkevich et al. [15] presented a methodology to enhance the stiffness analysis of serial and parallel manipulators with passive joints. The methodology directly takes into account the loading influence on the manipulator configuration as well as on its Jacobians and Hessians. Chakarov [16] discussed antagonistic stiffness of a parallel manipulator and showed that the maximum compliance in a random direction could be reduced by controlling the internal preload of linear actuators. Zhang and Gosselin [17] discussed a kinetostatic model for the Tricept machine tool based on lumped flexibility method. In their work, the optimization of the stiffness was performed using a genetic algorithm. Kim and Tsai [18] introduced a 3-DOF translational parallel manipulator, Cartesian parallel manipulator. An optimization method to maximize the stiffness is investigated in order to minimize the deflection at the joints caused by external forces exerted.

However, stiffness optimization is not a simple problem in redundantly actuated PKMs. It is not easy to intuitively understand the contributions of individual actuators towards improving active stiffness due to the coupling effect of internal preload torques in the active stiffness matrix in case of the redundantly actuated PKMs.

In this study, a decoupling method for the stiffness matrix is presented. The objective of the method is to solve mathematical problems in stiffness analysis and torque distributions of redundant PKM by decoupling its stiffness matrix to three (or more) linearly independent stiffness matrices of nonredundant cases to more clearly identify the contribution of individual actuators. One-redundant five-bar planar PKM is investigated. The PKM has two DOFs and three actuators for redundant actuation. Three actuating joints are decoupled into three groups containing two actuating joints. With this approach, the passive and active stiffness are analyzed and the analysis results are compared to original redundant cases.

This paper is organized as follows. In Section 2, kinematic configuration for the planar five-bar redundant parallel kinematic mechanism is introduced. Stiffness for the PKM is investigated in Section 3 with two cases: nonredundant and redundant cases. Decoupling of the kinematic stiffness matrix of the PKM is presented in Section 4. In Section 5, verification of the kinematic stiffness decoupling in the active and passive stiffness with various conditions is shown. Finally, concluding remarks are provided in Section 6.

\section{Kinematic Structure of Five-Bar Redundant Planar PKM}

The planar five-bar parallel kinematic mechanism is a wellknown PKM. As shown in Figure 1, it has a relatively simple kinematic structure comprising five bars (in other word, links), five joints, and one end-effector. Typically, the base frame is considered one bar.

Its DOF of end-effector are two and the required number of actuators at joints is also two. However, one or more actuators can be equipped with the remaining passive joints of the five-bar PKM to eliminate singularity and to increase stiffness. $L_{i}(i=1 \sim 5)$ indicates a bar, $A_{i}(i=1 \sim 5)$ indicates a joint, $\theta_{i}(i=1 \sim 4)$ is the joint angle, and $P$ is the end-effector. 
The left and right sides of $P$ construct two kinematic open chains. Except for the joint assigned to the end-effector, it is possible to attach four actuators to the four joints. As a result, up to two-redundant actuations can be implemented in this PKM.

\section{Stiffness of a Symmetric Five-Bar Planar Parallel Mechanism}

Stiffness can be defined as the ratio of the exerted force to an infinitesimal displacement. The stiffness matrix $K$ is composed of two parts. One is the active stiffness matrix and the other is the passive stiffness matrix. In this section, stiffness analyses for the nonredundant and redundant cases are presented.

\subsection{Nonredundant Case ( $q_{u}=A_{1}$ and $A_{2}$ Joints $)$}

3.1.1. Forward Kinematics and Jacobian. Forward kinematics is analyzed to determine the final position and orientation of the end-effector for given displacements of actuators. Forward kinematics is also applied to stiffness analysis. Forward Jacobian is included in both passive and active stiffness terms. $x$ - and $y$-coordinates of the end-effector position are represented by $A_{1}$ and $A_{2}$ joint angles:

$$
P(x, y)=\left[\begin{array}{l}
x \\
y
\end{array}\right]=\left[\begin{array}{c}
L_{1} C_{1}+L_{3} C_{13}-\frac{L_{5}}{2} \\
L_{1} S_{1}+L_{3} S_{13}
\end{array}\right] .
$$

In (1), $C_{1}$ and $C_{13}$ stand for shorthand of cosine theta 1 and cosine theta 1 plus theta 3 , respectively. This relationship is obtained from the left chain of the end-effector. By differentiation with respect to time, we get (2). $J_{f}$ is the forward Jacobian and it relates the velocity of the joint angle to the velocity of the end-effector position:

$$
\dot{P}=J_{f} \dot{\theta}
$$

3.1.2. Constraint Jacobians. The constraint Jacobian is obtained from the kinematic constraint equations. The kinematic constraints are established from the fact that the chain composed of five bars is a closed loop. It is assumed that $A_{1}$ and $A_{2}$ are assigned as actuation joints. The formulation of this constraint can be written as

$$
\begin{aligned}
G_{1}\left(\theta_{1}, \theta_{2}, \theta_{3}, \theta_{4}\right)= & L_{1} C_{1}+L_{3} C_{13}-L_{2} C_{2}-L_{4} C_{24} \\
& -L_{5}=0, \\
G_{2}\left(\theta_{1}, \theta_{2}, \theta_{3}, \theta_{4}\right)= & L_{1} S_{1}+L_{3} S_{13}-L_{2} S_{2}-L_{4} S_{24}=0 .
\end{aligned}
$$

Time derivative of (3) is shown in the following:

$$
\frac{d G}{d t}=\frac{d G}{d \theta} \frac{d \theta}{d t}=\frac{d G}{d \theta} \dot{\theta}=0
$$

The forward Jacobian is represented by (5). In detail, the forward Jacobian is composed of two terms. $\Phi$ is the constraint Jacobian:

$$
\begin{aligned}
J_{f}= & J_{u}+J_{v} \Phi \\
\Phi= & -G_{v}^{-1} G_{u} \in \mathfrak{R}^{2 \times 2}, \\
J_{u}= & {\left[\begin{array}{ll}
\frac{\partial P_{1}}{\partial \theta_{1}} & \frac{\partial P_{1}}{\partial \theta_{2}} \\
\frac{\partial P_{2}}{\partial \theta_{1}} & \frac{\partial P_{2}}{\partial \theta_{2}}
\end{array}\right], } \\
J_{v}= & {\left[\begin{array}{ll}
\frac{\partial P_{1}}{\partial \theta_{3}} & \frac{\partial P_{1}}{\partial \theta_{4}} \\
\frac{\partial P_{2}}{\partial \theta_{3}} & \frac{\partial P_{2}}{\partial \theta_{4}}
\end{array}\right], } \\
G_{u}= & {\left[\begin{array}{ll}
\frac{\partial G_{1}}{\partial \theta_{1}} & \frac{\partial G_{1}}{\partial \theta_{2}} \\
\frac{\partial G_{2}}{\partial \theta_{1}} & \frac{\partial G_{2}}{\partial \theta_{2}}
\end{array}\right], } \\
G_{v}= & {\left[\begin{array}{ll}
\frac{\partial G_{1}}{\partial \theta_{3}} & \frac{\partial G_{1}}{\partial \theta_{4}} \\
\frac{\partial G_{2}}{\partial \theta_{3}} & \frac{\partial G_{2}}{\partial \theta_{4}}
\end{array}\right] . }
\end{aligned}
$$

Because we selected $A_{1}$ and $A_{2}$ as the independent joints, the joint rearrangement matrix $U$ and the actuator selection matrix $V$ is represented as below:

$$
\begin{gathered}
q_{\text {all }}=U\left[\begin{array}{ll}
q_{u} & q_{v}
\end{array}\right]^{T} \Longrightarrow U_{12}=\left[\begin{array}{llll}
1 & 0 & 0 & 0 \\
0 & 1 & 0 & 0 \\
0 & 0 & 1 & 0 \\
0 & 0 & 0 & 1
\end{array}\right] \in \mathfrak{R}^{4 \times 4}, \\
q_{r}=V q_{\text {all }} \Longrightarrow V_{12}=\left[\begin{array}{llll}
1 & 0 & 0 & 0 \\
0 & 1 & 0 & 0
\end{array}\right] \in \mathfrak{R}^{2 \times 4}
\end{gathered}
$$

$q_{\text {all }}$ is joint value vector including all the joints. $q_{u}$ and $q_{v}$ are independent and dependent joint value vectors, respectively. $q_{r}$ is actuating joint value vector. $\Gamma$ is another constraint Jacobian containing $\Phi$ as the matrix element and it will be used in stiffness analysis. In the nonredundant case, however, $\Gamma$ is a two by two identity matrix as shown in the following:

$$
\begin{aligned}
\Gamma_{\text {all }} & =V U\left[\begin{array}{cc}
1 & 0 \\
0 & 1 \\
\Phi_{11} & \Phi_{12} \\
\Phi_{21} & \Phi_{22}
\end{array}\right]=\left[\begin{array}{llll}
1 & 0 & 0 & 0 \\
0 & 1 & 0 & 0
\end{array}\right]\left[\begin{array}{cc}
1 & 0 \\
0 & 1 \\
\Phi_{11} & \Phi_{12} \\
\Phi_{21} & \Phi_{22}
\end{array}\right] \\
& =\left[\begin{array}{ll}
1 & 0 \\
0 & 1
\end{array}\right] \in \mathfrak{R}^{2 \times 2} .
\end{aligned}
$$


3.1.3. Kinematic Stiffness Analysis with Hessian Matrix. The relationship between the actuating joint torque of PKMs and the force exerted on the end-effector of the mechanisms can be obtained by the virtual work theorem as in the following [19]:

$$
\begin{aligned}
\tau_{r}^{T} d q_{r}+f^{T} d x_{c} & =0, \\
\tau_{r}^{T} \Gamma d q_{u}+f^{T} J_{f} d q_{u} & =0, \\
f & =\left(\Gamma J_{f}^{-1}\right)^{T}\left(-\tau_{r}\right)=\Psi^{T}\left(-\tau_{r}\right),
\end{aligned}
$$

where $d x_{c}=J_{f} d q_{u}, d q_{r}=\Gamma d q_{u}, \Psi=\Gamma J_{f}^{-1}$.

In $(10), \tau_{r}$ is torque value vector of actuating joint. $f$ is exerted force and $x_{c}$ is end-effector position. Inverse of $J_{f}$ exists except for singularity position. Five-bar planar PKM has actuator and end-effector singularities. The actuator singularity is fully eliminated by the redundant actuation. Moreover, the end-effector is avoidable because it appeared only at the boundary of workspace. Therefore, it can be regarded that inverse of $J_{f}$ always exists. Stiffness can be defined as the ratio of the exerted force to an infinitesimal displacement:

$$
\begin{aligned}
K_{12} & =\frac{d f}{d x_{c}}=K_{\text {active }}+K_{\text {passive }} \\
& =H^{T} \otimes \tau_{u}+J_{f}^{-1 T} k_{u} J_{f}^{-1} .
\end{aligned}
$$

$H$ is Hessian matrix and $k_{u}$ is actuator stiffness matrix. A two-by-two stiffness matrix, $K_{12}$, which represents the stiffness matrix generated by actuating the $A_{1}$ and $A_{2}$ joints, is composed of active stiffness and passive stiffness as shown in (11). The first term of (11) is the active stiffness matrix and the second term is the passive stiffness matrix.

\subsection{Redundant Case ( $q_{u}=A_{1}, A_{2}$, and $A_{3}$ Joints $)$}

3.2.1. Constraint Jacobians. In this redundant case, $A_{1}, A_{2}$, and $A_{3}$ are assigned as actuation joints. The selection matrix $V$ is shown in (12) and the constraint Jacobian $\Gamma$ is different from the nonredundant case. The dimension of the selection matrix changes from two-by-four to three-by-four:

$$
q_{r}=V q_{\text {all }} \Longrightarrow V=\left[\begin{array}{llll}
1 & 0 & 0 & 0 \\
0 & 1 & 0 & 0 \\
0 & 0 & 1 & 0
\end{array}\right] \in \mathfrak{R}^{3 \times 4}
$$

Consequently, $\Gamma$ contains $\Phi_{11}$ and $\Phi_{12}$ as matrix elements as in (13). This change affects the stiffness matrix, which will be shown in the next sub sections:

$$
\Gamma=V U\left[\begin{array}{cc}
1 & 0 \\
0 & 1 \\
\Phi_{11} & \Phi_{12} \\
\Phi_{21} & \Phi_{22}
\end{array}\right]=\left[\begin{array}{llll}
1 & 0 & 0 & 0 \\
0 & 1 & 0 & 0 \\
0 & 0 & 1 & 0
\end{array}\right]\left[\begin{array}{cc}
1 & 0 \\
0 & 1 \\
\Phi_{11} & \Phi_{12} \\
\Phi_{21} & \Phi_{22}
\end{array}\right]
$$

$$
=\left[\begin{array}{cc}
1 & 0 \\
0 & 1 \\
\Phi_{11} & \Phi_{12}
\end{array}\right] \in \mathfrak{R}^{3 \times 2} .
$$

3.2.2. Kinematic Stiffness Analysis with Hessian Matrix. The subscript of $K_{123}$ in (14) represents the stiffness in the case of one-redundant, three-actuation. $\Psi$ is the product of the constraint Jacobian $\Gamma$ and the inverse of the forward Jacobian $J_{f}$ as $(10) .\left(\Gamma^{T}\right)^{+}$means the pseudoinverse of $\Gamma^{T}$ :

$$
\begin{aligned}
K_{123} & =\frac{d f}{d x_{c}}=K_{\text {active }}+K_{\text {passive }}=\frac{d\left(\Psi^{T}\left(-\tau_{r}\right)\right)}{d x_{c}} \\
& =\frac{d \Psi^{T}}{d x_{c}} \otimes\left(-\tau_{r}\right)+\Psi^{T} \frac{d\left(-\tau_{r}\right)}{d q_{r}} \frac{d q_{r}}{d x_{c}} \\
& =H^{T} \otimes \tau_{r}+\Psi^{T} k_{r} \Psi,
\end{aligned}
$$

where $d q_{r}=\Psi d x_{c} \in \mathfrak{R}^{2 \times 2}, \Psi=\Gamma J_{f}^{-1}, \Gamma^{T} \tau_{r}=\tau_{u}$, and $\tau_{r}=$ $\left(\Gamma^{T}\right)^{+} \tau_{u}+\left(I_{n}-\left(\Gamma^{T}\right)^{+} \Gamma^{T}\right) \varepsilon_{n}$.

In this case, the magnitude of active stiffness can be controlled by internal preload torque distribution. And the following equation is for finding the internal torque, which does not affect the motion of the five-bar planar PKM:

$$
\begin{aligned}
\Gamma^{T} \tau_{r} & =\left[\begin{array}{lll}
1 & 0 & \Phi_{11} \\
0 & 1 & \Phi_{12}
\end{array}\right]\left[\begin{array}{l}
\tau_{1} \\
\tau_{2} \\
\tau_{3}
\end{array}\right]=\left[\begin{array}{l}
0 \\
0
\end{array}\right] \Longrightarrow\left[\begin{array}{l}
\tau_{1}+\Phi_{11} \tau_{2} \\
\tau_{2}+\Phi_{12} \tau_{3}
\end{array}\right] \\
& =\left[\begin{array}{l}
0 \\
0
\end{array}\right] .
\end{aligned}
$$

As shown in Figure 2, $\tau_{r}$ contains not only internal preload torques that can increase the active stiffness of a oneredundant $\mathrm{PKM}$, but also the reactive (this case) or motional torques induced by the external forces. In addition, the PKM maintains static equilibrium state, although the internal preload torques are applied at all actuating joints. Then, additional torques, reactive torques, are generated by imposing the external force at the end-effector, and it is directly related to independent joint torques by Jacobian $\tau_{u}=-J^{T} f_{\text {ext }}$. As a 


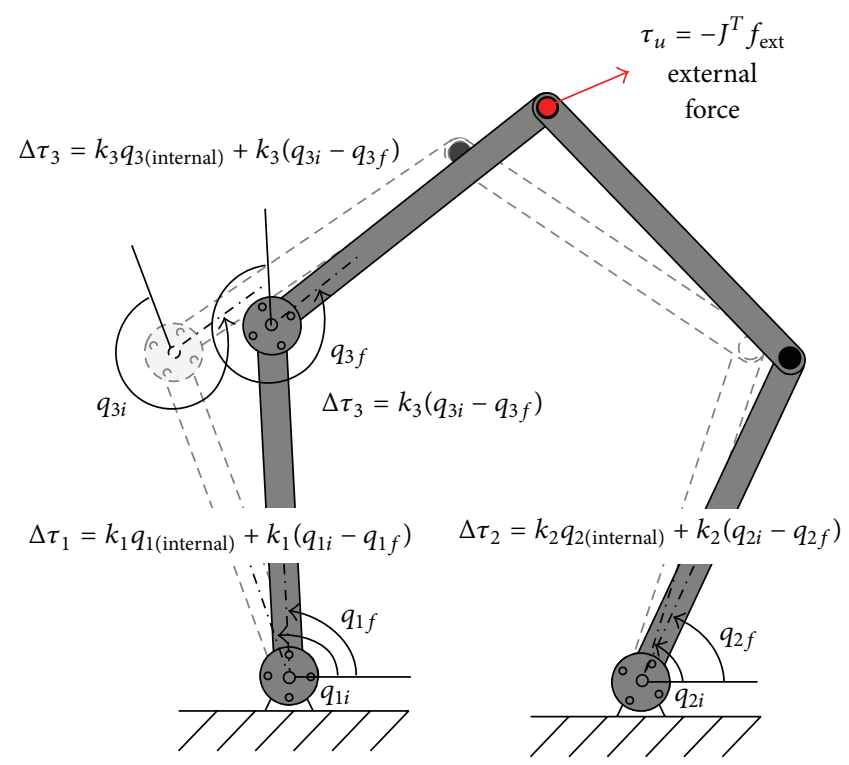

FIGURE 2: Reactive torque generated by external force exerted on the end-effector.

result, the stiffness matrix can be represented by the following equation for given external force:

$$
\begin{aligned}
K_{123}= & H^{T} \\
& \otimes(\underbrace{\left(\Gamma^{T}\right)^{+}\left(-J^{T} f_{\text {ext }}\right)}_{\tau_{r, \text { motional }}}+\underbrace{\left(I_{n}-\left(\Gamma^{T}\right)^{+} \Gamma^{T}\right) \varepsilon_{n}}_{\tau_{r, \text { internal }}}) \\
& +\Psi^{T} k_{r} \Psi .
\end{aligned}
$$

\section{Decoupling of the Kinematic Stiffness Matrix of the Redundant Five-Bar Planar PKM}

4.1. Multiplication of the Multidimensional Matrix: Hessian. In mathematics, the Jacobian matrix, which defines a linear map $\mathbb{R}^{n} \rightarrow \mathbb{R}^{m}$, is well known as the best linear approximation of the function $F$ near the point $x$. In the constraint Jacobian $\Phi$, the function $F$ denotes the velocity vector of the independent joint $q_{u}$ and the point $x$ denotes the velocity vector of the dependent joint $q_{v}$. Thus, this constraint Jacobian can be interpreted as the velocity relationship between the independent joint $q_{u}$ and the dependent joint $q_{v}$. This relationship can be obtained by the time derivative of the geometrical constraint equations. In a similar manner, the forward Jacobian matrix $J_{f}$ is also used to obtain the relationship between the active joint torque $\tau_{r}$ and the external force $f$ exerted at the end-effector.

The Hessian matrix is typically defined as a square matrix of the 2nd order partial derivatives of a scalar-valued function in mathematics. In this research, the derivative of the transposition of the inverse of the forward Jacobian $J_{f}$ with respect to the end-effector's position $x_{c}$ denotes the transposition of the

\begin{tabular}{|c|c|}
\hline Name & Operator and dimensions \\
\hline $\begin{array}{l}\text { Jacobian } \\
\text { matrix, } J\end{array}$ & $\begin{aligned} \frac{d Y}{d X}= & {\left[\begin{array}{lll}\frac{\partial Y}{\partial x_{1}} & \cdots & \frac{\partial Y}{\partial x_{n}}\end{array}\right] \in R^{m \times n} } \\
& \left\{\begin{array}{l}\frac{\partial Y}{\partial x_{1}} \in R^{m \times 1} \\
Y \in R^{m}\end{array}\right.\end{aligned}$ \\
\hline $\begin{array}{l}\text { Hessian } \\
\text { matrix, } H\end{array}$ & $\begin{aligned} \frac{d J}{d X^{\prime}}= & {\left[\begin{array}{lll}\frac{\partial J}{\partial x_{1}^{\prime}} & \cdots & \frac{\partial J}{\partial x_{k}^{\prime}}\end{array}\right] \in R^{m \times n \times k}, } \\
& \begin{cases}J \in R^{m \times n} \\
X^{\prime} \in R^{k}\end{cases} \end{aligned}$ \\
\hline $\begin{array}{l}\text { Leibniz's law } \\
\text { of } H \text { and } \\
\text { vector }\end{array}$ & $\begin{aligned} {[H \otimes \tau]=} & {\left[\begin{array}{ccc}\sum_{i=1}^{n} h_{1 i 1} \tau_{i 1} & \cdots & \sum_{i=1}^{n} h_{1 i k} \tau_{i 1} \\
\vdots & \ddots & \vdots \\
\sum_{i=1}^{n} h_{m i 1} \tau_{i 1} & \cdots & \sum_{i=1}^{n} h_{m i k} \tau_{i 1}\end{array}\right.} \\
& \left\{\begin{array}{l}H \in R^{m \times n \times k} \\
\tau \in R^{n \times 1}\end{array}\right.\end{aligned}$ \\
\hline
\end{tabular}
Hessian matrix $H$. Physically, the derivative of the Jacobian
TABLE 1: Mathematical definitions and notations.

matrix is related to the stiffness matrix and the active torque. Nakamura and Ghodoussi [20] defined the Hessian matrix and suggested the operating method of the Hessian matrix and vector. The result of Leibniz's law of the Hessian matrix and the active torque vector can be defined as the active stiffness of the mechanism [2].

The detailed definitions of the Jacobian matrix $J$, Hessian matrix $H$, and Leibniz's law are represented as shown in Table 1. 


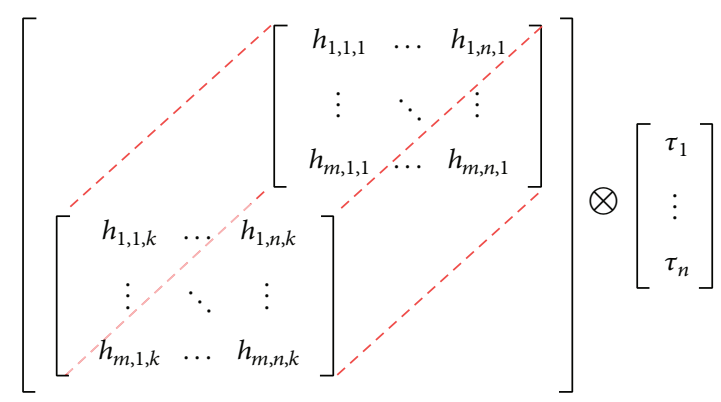

FIGURE 3: The schematic of the multiple two-dimensional matrices consisting of the Hessian matrix and vector.

Typically, a matrix with more than two dimensions is called a multidimensional matrix. An element of the twodimensional matrix can be accessed with two subscripts. The first subscript denotes the row index, and the second subscript denotes the column index. However, the multidimensional matrix uses an additional subscript for additional indexing. Figure 3 represents the concept of multiple twodimensional matrices of three and higher dimensions.

The proposed Hessian matrix $H$ of the forward Jacobian $J_{f}$ is a three-dimensional $m \times n \times k$ matrix consisting of $k$ pages of $m \times n$ matrix as shown in Figure 3. To compute the stiffness matrix, Leibniz's law of the Hessian matrix $H$ and the active torque vector $\tau_{r}$ should be calculated by using the operator $\otimes$ as shown below:

$$
\begin{aligned}
K & =\frac{d\left(J_{f}^{-1}\right)^{T}\left(-\tau_{r}\right)}{d x_{c}} \\
& =\frac{d\left(J_{f}^{-1}\right)^{T}}{d q_{r}} J_{f}^{-1} \otimes \tau_{r}+\left(J_{f}^{-1}\right)^{T} \frac{d\left(-\tau_{r}\right)}{d q_{r}} J_{f}^{-1} \\
& =H^{T} \otimes \tau_{r}+\left(J_{f}^{-1}\right)^{T} k_{r} J_{f}^{-1}, \quad\left(\because \frac{d q_{r}}{d x_{c}}=J_{f}^{-1}\right) .
\end{aligned}
$$

For nonredundant cases of the five-bar planar PKM,

$$
K_{\text {active }}=H^{T} \otimes \tau_{u} \in R^{2 \times 2},
$$

where $\tau_{u}=V_{\tau}\left[\begin{array}{lll}\tau_{1} & \tau_{2} & \tau_{3}\end{array}\right]^{T} \in R^{2 \times 1}, H^{T}=\left(d\left(J_{f}^{-1}\right)^{T} / d q_{u}\right) J_{f}^{-1} \in$ $R^{2 \times 2 \times 2}$, and $q_{u}=V_{q}\left[\begin{array}{llll}q_{1} & q_{2} & q_{3} & q_{4}\end{array}\right]^{T} \in R^{2 \times 1}$.

For redundant cases of one more actuation for the five-bar planar PKM,

$$
K_{\text {active }}=H^{T} \otimes \tau_{r} \in R^{2 \times 2},
$$

where $\tau_{r}=V_{\tau}\left[\begin{array}{lll}\tau_{1} & \tau_{2} & \tau_{3}\end{array}\right]^{T} \in R^{3 \times 1}, H^{T}=\left(d\left(J_{f}^{-1}\right)^{T} / d q_{r}\right) J_{f}^{-1} \in$ $R^{2 \times 3 \times 2}$, and $q_{r}=V_{q}\left[\begin{array}{llll}q_{1} & q_{2} & q_{3} & q_{4}\end{array}\right]^{T} \in R^{3 \times 1}$.

\subsection{Stiffness Decoupling for Nonredundant and \\ Redundant Five-Bar Planar PKM}

4.2.1. Active Stiffness. It should be noted that all of the actuating joints $q_{r}$ in the redundant actuation can be decoupled to three or more groups containing two independent joints $q_{u}$ of the nonredundant actuations in the stiffness analysis as shown in Figure 4. This means that the total sum of the active stiffness matrices in all possible groups of nonredundant actuations is exactly equal to a resultant active stiffness matrix of the redundant actuation:

$$
\begin{aligned}
K_{\text {active, redundant }} & =\sum_{\{s \in U\}} H_{s}^{T} \otimes \tau_{s} \\
& =\left(\left(\frac{\partial \Psi^{T}}{\partial q_{u}}+\frac{\partial \Psi^{T}}{\partial q_{v}} \Phi\right) J_{f}^{-1}\right) \otimes\left[\begin{array}{c}
\tau_{1} \\
\tau_{2} \\
\tau_{3}
\end{array}\right],
\end{aligned}
$$

where $\sum_{\{s \in U\}} H_{s}^{T} \otimes \tau_{s}=\left[H_{12}^{T} \otimes \tau_{12}+H_{23}^{T} \otimes \tau_{23}+H_{31}^{T} \otimes \tau_{31}\right]$, $H_{12}^{T} \equiv\left(\partial\left(J_{12}^{-1}\right)^{T} / \partial q_{12}+\left(\partial\left(J_{12}^{-1}\right)^{T} / \partial q_{34}\right) \Phi_{12}\right) J_{12}^{-1}, \tau_{12}=\left[\begin{array}{ll}\tau_{1} & \tau_{2}\end{array}\right]^{T}$, $q_{u}=\left[\begin{array}{lll}q_{1} & q_{2} & q_{3}\end{array}\right]^{T}$, and $q_{v}=q_{4}$.

First of all, the nonredundant five-bar planar PKM can fully manipulate the position of the platform in the $x y$-plane by using two active joints; thus the required number of active joints should be two. With one more actuator assigned as an additional active joint, it is possible that the magnitude of active stiffness of the five-bar planar PKM can be changed by the torque distributions, because there are infinite numbers of possible combinations of the active joints' torque-like indeterminate forms. Physically, there are infinite numbers of sets of torque distributions for individual active joints even though all motions in the platform, links, and joints are the same. So, with three active joints for the one-redundant five-bar planar PKM, there must exist three combinations of nonredundant actuation as shown in (20). The summation index is an element $s$ of the set $U=\{(1,2),(2,3)$, $(3,1)\}$.

From the perspective of stiffness decoupling with redundancy in the torque distribution, (21) represents the calculation formula for the active stiffness according to oneredundant actuation by using $\left[q_{1}, q_{2}, q_{3}\right]^{T}$. The first and second equations in (21) show that the sum of the individual active stiffness matrices of nonredundant actuations should be equal to a resultant active stiffness matrix of the oneredundant actuation. In addition, the distribution of three active torques for individual nonredundant actuation can vary in the range that meets the given constraint related to torque distribution. That is, the active joint torques calculated by the torque distribution can be successfully reallocated 


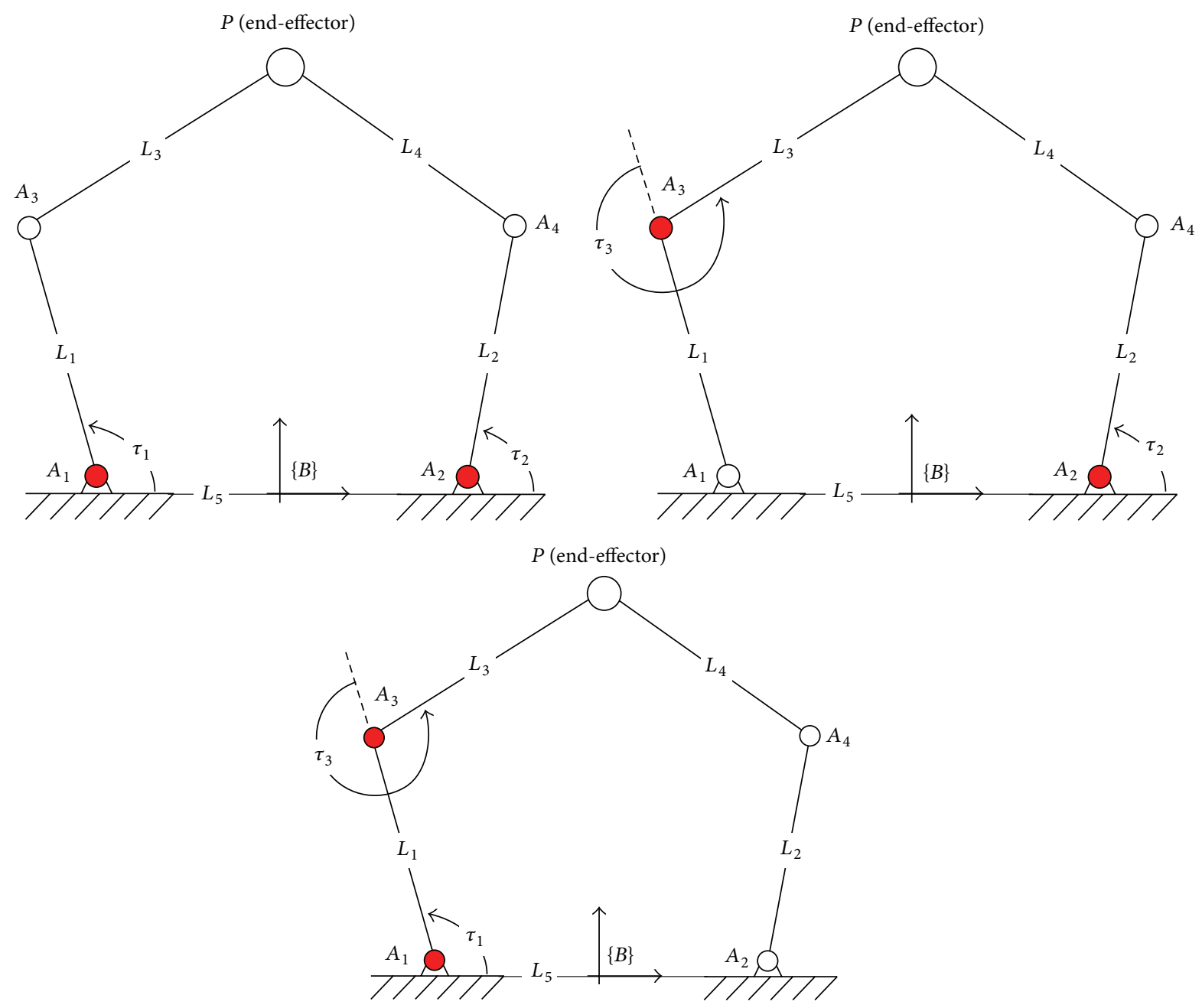

FIgURE 4: Schematic diagram of the three-independent joint set.

to individual nonredundant actuation. In particular, mathematical sense becomes more apparent in the third equation. The matrix equations can be interpreted as linear combinations of three active torque vectors:

$$
K_{\text {active, redundant }}=\left(\left(\frac{\partial \Psi^{T}}{\partial q_{u}}+\frac{\partial \Psi^{T}}{\partial q_{v}} \Phi\right) J_{f}^{-1}\right) \otimes\left[\begin{array}{l}
w_{1} \tau_{1} \\
w_{2} \tau_{2} \\
w_{3} \tau_{3}
\end{array}\right]
$$

Nonredundant

$$
=\left\{\begin{array}{r}
H_{12}^{T} \otimes\left[\begin{array}{c}
w_{1,1} \tau_{1} \\
w_{2,1} \tau_{2}
\end{array}\right]+H_{23}^{T} \otimes\left[\begin{array}{c}
w_{2,2} \tau_{2} \\
w_{3,1} \tau_{3}
\end{array}\right]+H_{31}^{T} \otimes\left[\begin{array}{c}
w_{3,2} \tau_{3} \\
w_{1,2} \tau_{1}
\end{array}\right] \\
H_{12}^{T} \otimes\left[\begin{array}{c}
w_{1} \tau_{1} \\
0
\end{array}\right]+H_{23}^{T} \otimes\left[\begin{array}{c}
w_{2} \tau_{2} \\
0
\end{array}\right]+H_{31}^{T} \otimes\left[\begin{array}{c}
w_{3} \tau_{3} \\
0
\end{array}\right], \\
\text { where, } w_{i}=\sum_{j=1}^{2} w_{i, j} .
\end{array}\right.
$$

Since each element of the Hessian matrix can be considered a constant matrix, the resultant active stiffness matrix of one-redundant actuation can be obtained by superposition of three linearly independent active stiffness matrices in nonredundant actuations. Finally, this explicit stiffness decoupling method using the third equation of (21) allows us to understand which active joint's torque is more sensitive and contributes the most to improving and controlling the magnitude and isotropy of the active stiffness of one- or two-redundant actuation without using the matrix with the complex coupling effects of all internal torques.

4.2.2. Passive Stiffness. Passive stiffness of the one-redundant actuation PKM can also be decoupled into three groups containing two-torsional stiffness of the active actuators as shown in (22). The passive stiffness is composed of the actuators' torsional stiffness matrix, the product of the constraint Jacobian $\Gamma$, and the inverse of the forward Jacobian $J_{f}$ as in (14). That is, the resultant passive stiffness matrix is a constant if the position and orientation of the platform of PKM and the actuators' torsional stiffness are determined. The passive stiffness matrix is perfectly independent because it is not dependent on internal preload torques. 
Finally, using this stiffness decoupling method allows us to figure out which torsional stiffness of the active actuators is more sensitive and contributes most to improving and controlling the magnitude and isotropy of the total stiffness of one- or two-redundant actuation without using the matrix with the complex coupling effects of all torsional stiffness and kinematic parameters:

$$
K_{\text {passive, redundant }}=\Psi^{T}\left[\begin{array}{ccc}
\left(w_{1,1}+w_{1,2}\right) k_{1} & 0 & 0 \\
0 & \left(w_{2,1}+w_{2,2}\right) k_{2} & 0 \\
0 & 0 & \left(w_{3,1}+w_{3,2}\right) k_{3}
\end{array}\right] \Psi
$$

Nonredundant

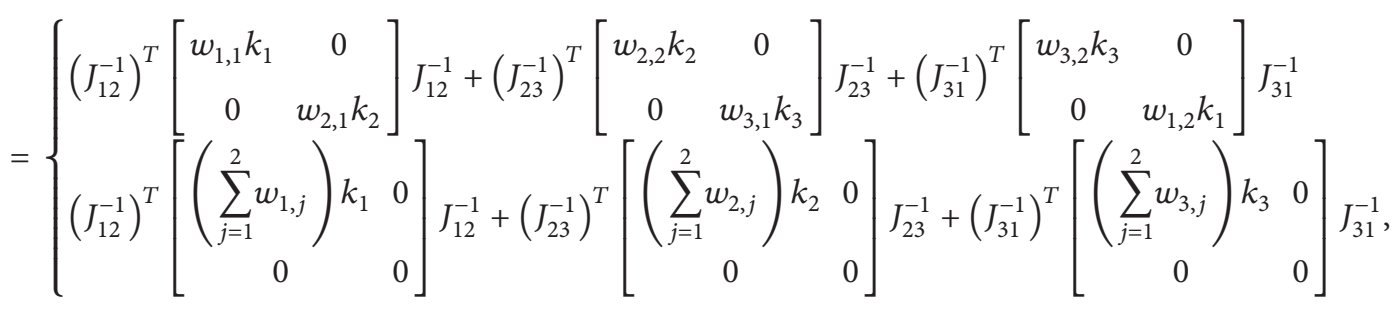

$$
\begin{aligned}
& \text { where, } w_{i}=\sum_{j=1}^{2} w_{i, j}, k_{i} \text { : actuators' torsional stiffness. }
\end{aligned}
$$

\section{Verification of the Stiffness Decoupling Method}

In this section, the proposed stiffness decoupling method is fully verified by applying distributed internal preload torques for the one-redundant actuation of five-bar planar PKM to its three groups of nonredundant actuation cases as represented in (21) and (22).

As mentioned earlier, the active stiffness of a redundant PKM can be changed by not only the motional torques but also the internal preload torques. That is, the torques distributed to calculate the active stiffness should not be the motional torques, but the internal preload torques. Thus, this can be obtained from the following relationship in case of the one-redundant actuation:

$$
\begin{array}{r}
\Gamma^{T} \tau_{r}=\left[\begin{array}{lll}
1 & 0 & \Phi_{11} \\
0 & 1 & \Phi_{12}
\end{array}\right]\left[\begin{array}{l}
\tau_{1} \\
\tau_{2} \\
\tau_{3}
\end{array}\right]=\left[\begin{array}{l}
0 \\
0
\end{array}\right], \\
\tau_{1}=-\Phi_{11} \tau_{3}, \tau_{2}=-\Phi_{12} \tau_{3} .
\end{array}
$$

To verify the proposed explicit stiffness decoupling method, the internal preload torque of the first active joint is set to fifty percent of its maximum torque after gear reduction with the motional torque taken into consideration, and then the second and third active torque are obtained from the relationship of (23). As a result, the distributed joint torques, $\tau_{1}, \tau_{2}$, and $\tau_{3}$, are successfully obtained as shown in Table 2 .

The active stiffness of the one-redundant actuation is calculated by substituting the torques in the first equation of
(21) to the $\tau_{1}, \tau_{2}$, and $\tau_{3}$ as the conventional method. And, as shown in Table 3, it is confirmed that the resultant active stiffness calculated from the second and third equations of (21) is exactly equal to the active stiffness of the oneredundant actuation for two cases. Similarly, in case of the passive stiffness, it is also confirmed that the resultant passive stiffness is calculated from the first, second, and third equations of (22).

For all cases, it is confirmed that the total sum of decoupled three active and passive stiffness matrices is exactly equal to the stiffness matrices of one-redundant actuation, respectively. From this result, the contributions of all individual internal preload torques and torsional stiffness of the three active stiffness matrices in the total stiffness analysis can be intuitively discovered.

We performed additional simulations for verification of case-I by applying commercial dynamics simulation software, DAFUL. DAFUL implements a dynamic analysis algorithm based on the implicit method. According to the proposed theorem, the five-bar planar PKM will maintain static equilibrium only if external forces exerted on the end-effector and actuation torques are related by forward Jacobian, $J_{f}$. The three decoupled groups are put under the actuation torques calculated from internal preload torque distribution and corresponding external force as shown in Table 5. All kinematic parameters are set to be the same as $1-\mathrm{m}$ and the position of end-effector is set to be [0-m, 1.5388-m]. As shown in Figure 5, the simulation results show that the $x$ - and $y$ axis position of the end-effector does not change because the external forces applied at the end-effector and the torques applied at actuating joints are in static equilibrium. Three 

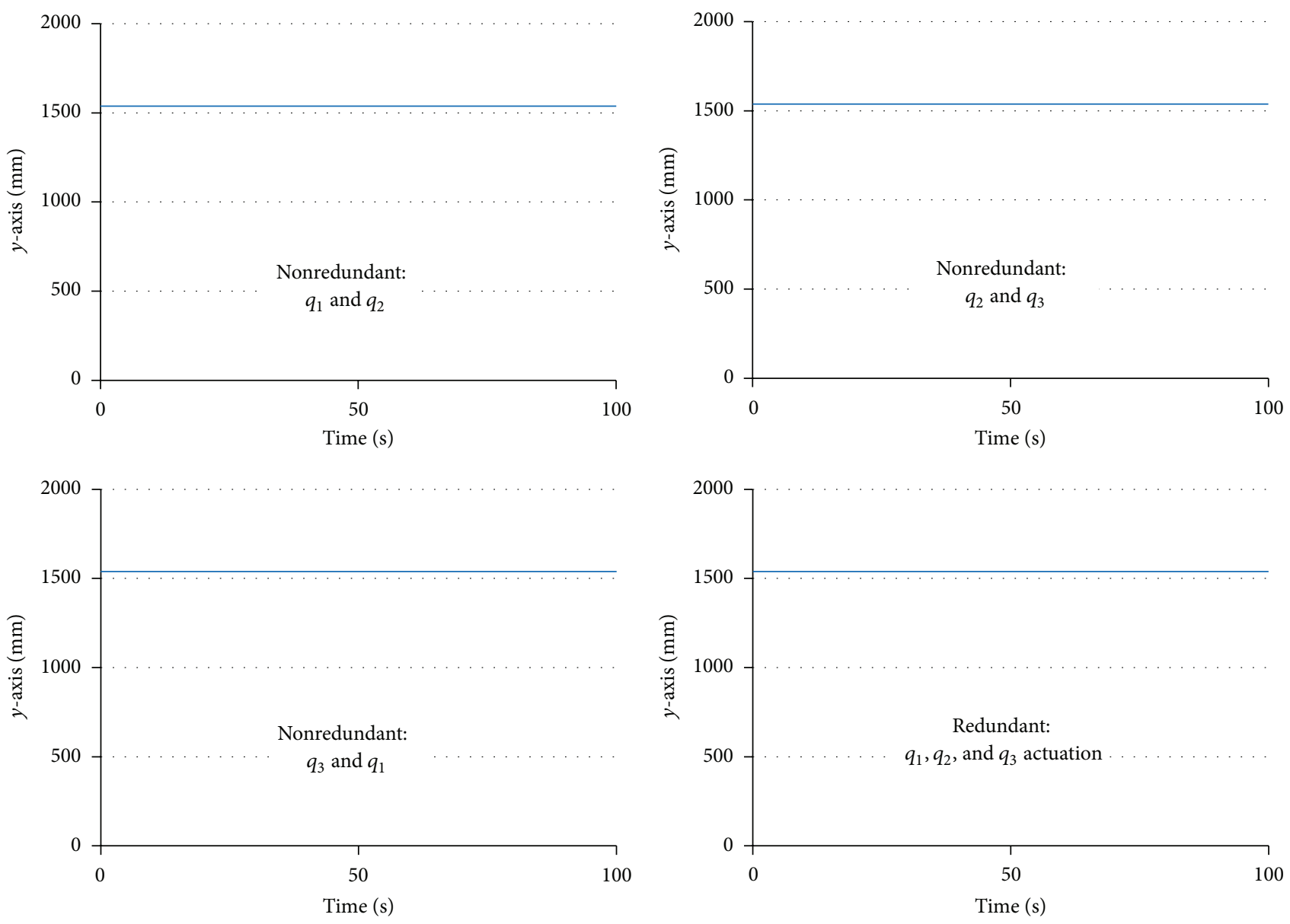

FIGURE 5: Verification results obtained from DAFUL simulation.

TABLE 2: Simulation conditions for the stiffness decoupling.

\begin{tabular}{|c|c|c|}
\hline Parameters & Case-1 & Case-2 \\
\hline Kinematic parameters [m] & \multicolumn{2}{|c|}{$[1,1,1,1,1]$} \\
\hline \multicolumn{3}{|l|}{ Weight factors } \\
\hline$w_{1,1}, w_{1,2}$ & {$\left[\begin{array}{ll}0.25 & 0.75\end{array}\right]$} & {$\left[\begin{array}{ll}1 & 0\end{array}\right]$} \\
\hline$w_{2,1}, w_{2,2}$ & {$\left[\begin{array}{ll}0.75 & 0.25\end{array}\right]$} & {$\left[\begin{array}{ll}0 & 1\end{array}\right]$} \\
\hline$w_{3,1}, w_{3,2}$ & {$\left[\begin{array}{ll}0.25 & 0.75\end{array}\right]$} & {$\left[\begin{array}{ll}1 & 0\end{array}\right]$} \\
\hline Joints' torsional stiffness & \multirow{2}{*}{\multicolumn{2}{|c|}{$\left[k_{1}, k_{2}, k_{3}\right]=[10,10,10]$}} \\
\hline Joints' active torques $[\mathrm{N} \cdot \mathrm{m}]$ & & \\
\hline$\tau_{1}$ & \multicolumn{2}{|c|}{65.00} \\
\hline$\tau_{2}$ & \multicolumn{2}{|c|}{-51.22} \\
\hline$\tau_{3}$ & \multicolumn{2}{|c|}{26.27} \\
\hline
\end{tabular}

decoupled nonredundant cases and redundant case (rightlower case in Figure 5) show the same $y$-axis position. These results mean that the proposed decoupling method turned out to be valid in mathematical and mechanical points of view.

\section{Conclusions}

Although the kinematic stiffness is not the actual stiffness of PKM, numerous researches have already proved that the
TABLE 3: Simulation results of the active stiffness decoupling.

\begin{tabular}{lccc}
\hline Kinds of actuation and & \multicolumn{3}{c}{ Elements of stiffness matrices $[\mathrm{N} / \mathrm{m}]$} \\
cases & $k_{11}$ & $k_{12}, k_{21}$ & $k_{22}$ \\
\hline $\begin{array}{l}\text { Nonredundant } \\
q_{1} \text { and } q_{2} \text { actuation }\end{array}$ & & & \\
$\quad$ Case-1 & -34.7261 & 6.6080 & -149.5999 \\
$\quad$ Case-2 & -41.2902 & -19.3766 & -177.8776 \\
Nonredundant & & & \\
$q_{2}$ and $q_{3}$ actuation & & & \\
$\quad$ Case-1 & 2.3697 & 11.1586 & -1.2604 \\
$\quad$ Case-2 & -32.5381 & 15.2695 & -140.1739 \\
Nonredundant & & & \\
$q_{3}$ and $q_{1}$ actuation & & & \\
$\quad$ Case-1 & & & \\
$\quad$ Case-2 & 0.5451 & 7.4912 & -32.0588 \\
Redundant actuation & 42.0169 & 29.3649 & 135.1325 \\
$\quad$ Case-1 & & & \\
$\quad$ Case-2 & -31.8114 & 25.2578 & -182.9190 \\
\hline
\end{tabular}

kinematic stiffness is worthy in design, analysis, and optimization processes for the PKMs. Thus, this paper describes a new explicit stiffness decoupling method for kinematic stiffness analysis of kinematically redundant PKM with twoDOF and one-redundant five-bar planar PKM. The stiffness 
TABLE 4: Simulation results of the passive stiffness decoupling.

\begin{tabular}{lcc}
\hline $\begin{array}{l}\text { Kinds of actuation and } \\
\text { cases }\end{array}$ & $k_{11}$ & Elements of stiffness matrices [N/m] \\
\hline $\begin{array}{l}\text { Nonredundant } \\
q_{1} \text { and } q_{2} \text { actuation }\end{array}$ & & $k_{12}, k_{21}$ \\
$\quad$ Case-1 & 7.2688 & -4.0493 \\
$\quad$ Case-2 & 7.2688 & 8.0986 \\
Nonredundant & & \\
$q_{2}$ and $q_{3}$ actuation & & \\
Case-1 & 2.8354 & 1.4985 \\
$\quad$ Case-2 & 7.2688 & -8.0986 \\
Nonredundant & & \\
$q_{3}$ and $q_{1}$ actuation & & \\
$\quad$ Case-1 & & \\
$\quad$ Case-2 & 8.5063 & 16.6434 \\
Redundant actuation & 4.0730 & 14.0925 \\
$\quad$ Case-1 & & \\
$\quad$ Case-2 & 18.6106 & 14.0231 \\
\end{tabular}

TABLE 5: Detailed conditions and results of verification for case-I of active stiffness decoupling.

\begin{tabular}{lccc}
\hline Actuating joints & $\begin{array}{c}\text { Actuating torques }[\mathrm{Nm}] \\
{\left[\tau_{1}, \tau_{2}, \tau_{3}\right]}\end{array}$ & $\begin{array}{c}\text { External forces }[\mathrm{N}] \\
{\left[f_{x}, f_{y}\right]}\end{array}$ & $\begin{array}{c}\text { End-effector position [m] } \\
{[x, y]}\end{array}$ \\
\hline $\begin{array}{l}\text { Nonredundant } \\
{\left[q_{1} \text { and } q_{2}\right]}\end{array}$ & {$\left[0.25 \cdot \tau_{1}, 0.75 \cdot \tau_{2}\right]$} & {$[18.86,-33.78]$} & {$[0,1.5388]$} \\
$\begin{array}{l}\text { Nonredundant } \\
{\left[q_{2} \text { and } q_{3}\right]}\end{array}$ & {$\left[0.25 \cdot \tau_{2}, 0.75 \cdot \tau_{3}\right]$} & {$[21.25,23.97]$} & {$[0,1.5388]$} \\
Nonredundant & {$\left[0.25 \cdot \tau_{3}, 0.75 \cdot \tau_{1}\right]$} & {$[-38.02,-19.50]$} & {$[0,1.5388]$} \\
{$\left[q_{3}\right.$ and $\left.q_{1}\right]$} & {$\left[\tau_{1}, \tau_{2}, \tau_{3}\right]$} & {$[2.09,-29.32]$} & {$[0,1.5388]$} \\
Redundant actuation & & & \\
\hline
\end{tabular}

Distributed joints' torques $\left[\tau_{1}, \tau_{2}, \tau_{3}\right]=[65.00,-51.22,26.27]$.

End-effector's position means resultant position when the joint torques and external forces of each case are applied to the PKM, simultaneously.

analyses for two cases are performed: nonredundant case composed of three nonredundant groups and one redundant case. For the nonredundant case, three actuating joints for the one-redundant actuation are decoupled to three groups to realize nonredundant actuation containing two actuating joints. Consequently, it is confirmed that the active and passive stiffness of one-redundant PKM are exactly equal to the total sum of active and passive stiffness of three nonredundant PKMs.

With this mathematical configuration, the contribution of each actuator can be intuitively investigated. For comparison, elements of stiffness matrices for the one-redundant and decoupled three nonredundant cases are listed in Tables 3 and 4 . Moreover, simulation results by applying the dynamic simulation tool are presented. This decoupling method is applicable to redundant PKMs with many passive joints. Selecting the optimal joint for redundant actuation from various candidates is more intuitive using this method.

\section{Conflict of Interests}

The authors declare that there is no conflict of interests regarding the publication of this paper.

\section{Acknowledgment}

This research was supported by Basic Science Research Program through National Research Foundation of Korea (NRF) funded by ministry of education (2014R1A1A2055334).

\section{References}

[1] S. Kim, W. In, H. Yim, J. I. Jeong, F. C. Park, and J. Kim, "Stiffness enhancement of a redundantly actuated parallel manipulator using internal preload: application to a 2-d.o.f parallel mechanism," in Proceedings of the Asian Symposium for Precision Engineering and Nanotechnology, pp. 6-9, 2007.

[2] S. Lee, S. Kim, W. In, M. Kim, J. I. Jeong, and J. Kim, "Experimental verification of antagonistic stiffness planning for a planar parallel mechanism with 2-DOF force redundancy," Robotica, vol. 29, no. 4, pp. 547-554, 2011.

[3] H. Shin, S. Lee, J. I. Jeong, and J. Kim, "Antagonistic stiffness optimization of redundantly actuated parallel manipulators in a predefined workspace," IEEE/ASME Transactions on Mechatronics, vol. 18, no. 3, pp. 1161-1169, 2013.

[4] S. J. Ryu, J. W. Kim, J. C. Hwang et al., "Eclipse: an overactuated parallel mechanism for rapid machining," in Parallel Kinematic 
Machines, Theoretical Aspects and Industrial Requirements, pp. 441-455, Springer, London, UK, 1999.

[5] A. Müller, "Internal preload control of redundantly actuated parallel manipulators-its application to backlash avoiding control," IEEE Transactions on Robotics, vol. 21, no. 4, pp. 668677,2005

[6] L. Wang, J. Wu, J. Wang, and Z. You, "An experimental study of a redundantly actuated parallel manipulator for a 5-DOF hybrid machine tool," IEEE/ASME Transactions on Mechatronics, vol. 14, no. 1, pp. 72-81, 2009.

[7] S. C. Lee, Research on antagonistic stiffness for over-actuated parallel mechanisms [Ph.D. thesis], School of Mechanical and Aerospace Engineering, Seoul National University, Seoul, South Korea, 2008.

[8] H. Shin, S. Kim, J. I. Jeong, and J. Kim, "Stiffness enhancement of a redundantly actuated parallel machine tool by dual support rims," International Journal of Precision Engineering and Manufacturing, vol. 13, no. 9, pp. 1539-1547, 2012.

[9] M. Arsenault and R. Boudreau, "Synthesis of planar parallel mechanisms while considering workspace, dexterity, stiffness and singularity avoidance," Journal of Mechanical Design, vol. 128, no. 1, pp. 69-78, 2005.

[10] G. Alici and B. Shirinzadeh, "Enhanced stiffness modeling, identification and characterization for robot manipulators," IEEE Transactions on Robotics, vol. 21, no. 4, pp. 554-564, 2005.

[11] X.-J. Liu, J. Wang, and G. Pritschow, "Performance atlases and optimum design of planar 5R symmetrical parallel mechanisms," Mechanism and Machine Theory, vol. 41, no. 2, pp. 119144,2006

[12] J. H. Lee, B.-J. Yi, S.-R. Oh, and I. H. Suh, "Optimal design of a five-bar finger with redundant actuation," in Proceedings of the IEEE International Conference on Robotics \& Automation, pp. 2068-2074, IEEE, Leuven, Belgium, May 1998.

[13] S. Kock and W. Schumacher, "A parallel x-y manipulator with actuation redundancy for high-speed and active-stiffness applications," in Proceedings of the IEEE International Conference on Robotics and Automation, vol. 3, pp. 2295-2300, IEEE, Leuven, Belgium, May 1998.

[14] Y. Li and Q. Xu, "Stiffness analysis for a 3-PUU parallel kinematic machine," Mechanism and Machine Theory, vol. 43, no. 2, pp. 186-200, 2008.

[15] A. Pashkevich, A. Klimchik, and D. Chablat, "Enhanced stiffness modeling of manipulators with passive joints," Mechanism and Machine Theory, vol. 46, no. 5, pp. 662-679, 2011.

[16] D. Chakarov, "Study of the antagonistic stiffness of parallel manipulators with actuation redundancy," Mechanism and Machine Theory, vol. 39, no. 6, pp. 583-601, 2004.

[17] D. Zhang and C. M. Gosselin, "Kinetostatic analysis and design optimization of the Tricept machine tool family," Journal of Manufacturing Science and Engineering-Transactions of the ASME, vol. 124, no. 3, pp. 725-733, 2002.

[18] H. S. Kim and L.-W. Tsai, "Design optimization of a cartesian parallel manipulator," Journal of Mechanical Design, vol. 125, no. 1, pp. 43-51, 2003.

[19] H. Shin, S. Lee, W. In, J. I. Jeong, and J. Kim, "Kinematic optimization of a redundantly actuated parallel mechanism for maximizing stiffness and workspace using Taguchi method," Journal of Computational and Nonlinear Dynamics, vol. 6, no. 1, Article ID 011017, 2011.
[20] Y. Nakamura and M. Ghodoussi, "Dynamics computation of closed-link robot mechanisms with nonredundant and redundant actuators," IEEE Transactions on Robotics and Automation, vol. 5, no. 3, pp. 294-302, 1989. 


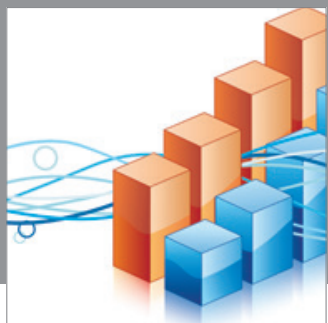

Advances in

Operations Research

mansans

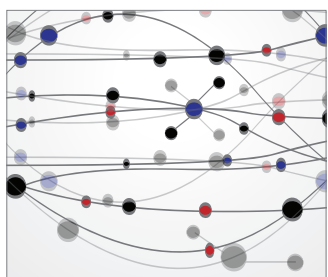

The Scientific World Journal
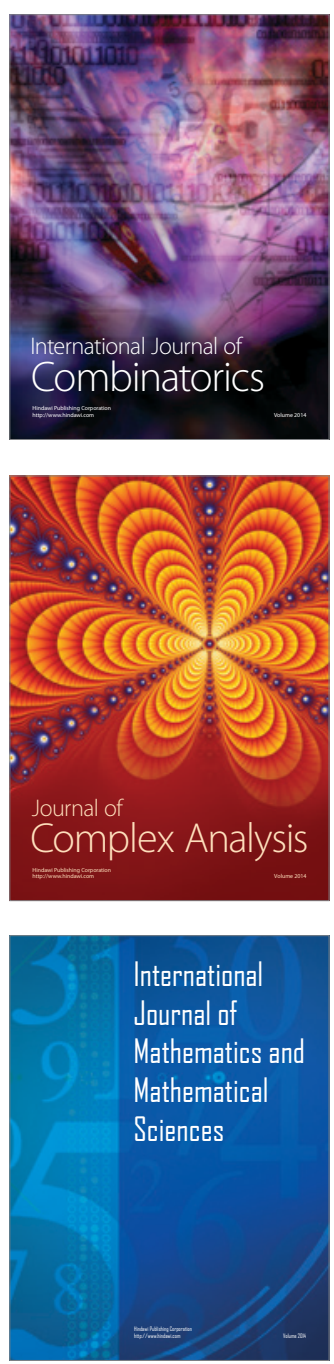
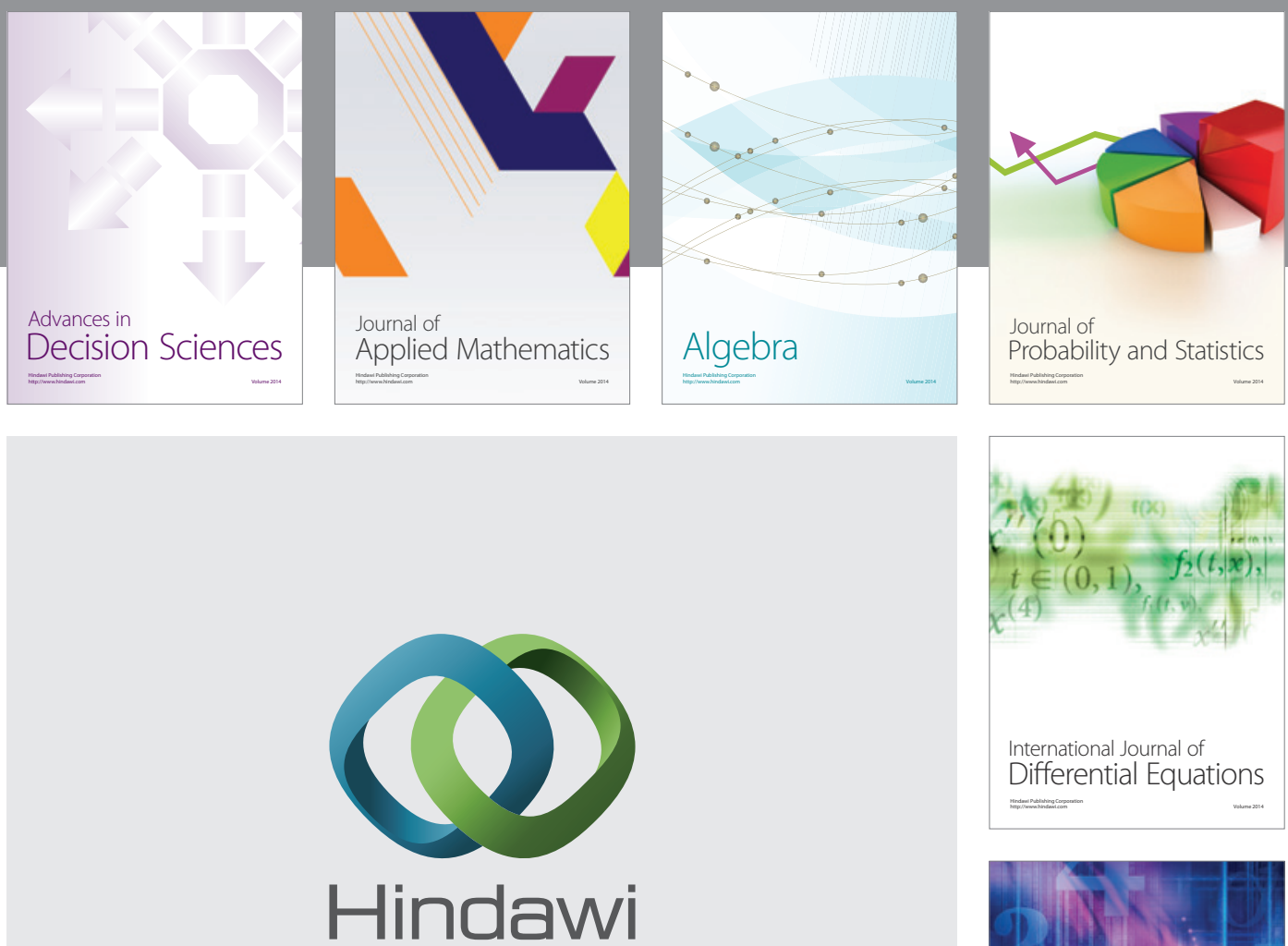

Submit your manuscripts at http://www.hindawi.com
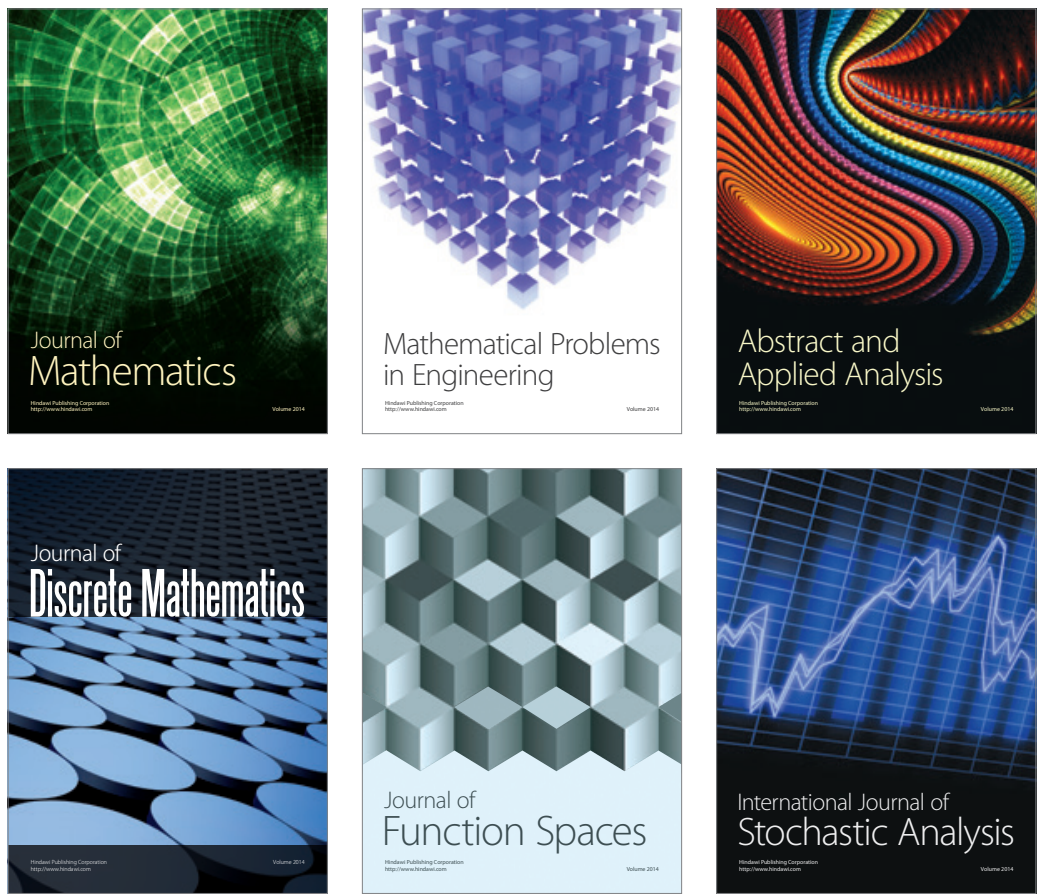

Journal of

Function Spaces

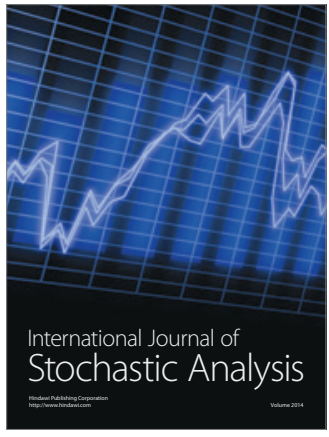

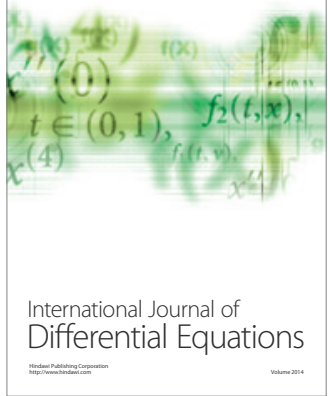
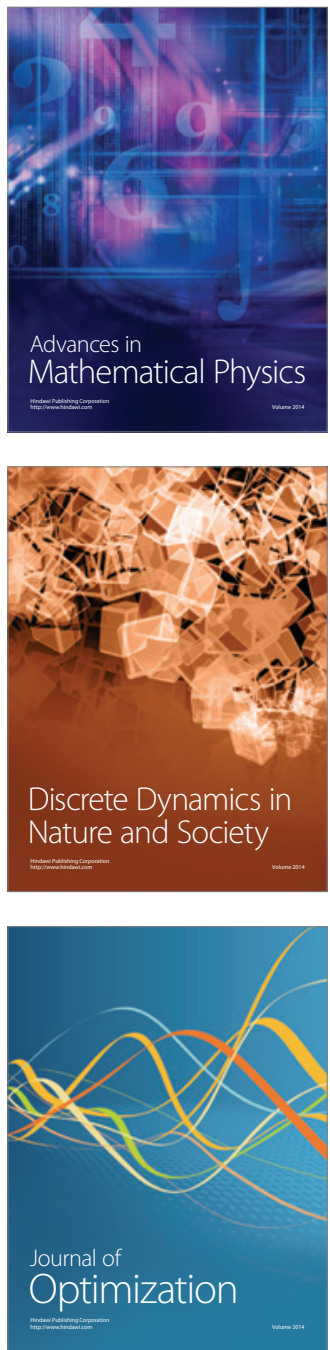